\title{
Nonlinear analysis of an electrodynamic broadband energy harvester
}

\author{
S. Bradai ${ }^{1,2, a}$, S. Naifar ${ }^{1,2}$, C. Viehweger ${ }^{1}$, O. Kanoun ${ }^{1}$, and G. Litak ${ }^{3,4}$
}

1 Chair for Measurement and Sensor Technology, Technische Universität Chemnitz, Reichenhainer Straße 70, 09126 Chemnitz, Germany

2 Laboratory of Electromechanical Systems, National Engineering School of Sfax, University of Sfax, Route de Soukra km 4, 3038 Sfax, Tunisia

3 Faculty of Mechanical Engineering, Lublin University of Technology, 20-618 Lublin, Poland

4 Laboratoire de Génie Electrique et Ferroélectricité, Institut National des Sciences Appliquées de Lyon, 8 rue de la Physique, 69621 Villeurbanne Cedex, France

Received 25 August 2015 / Received in final form 9 September 2015 Published online 20 November 2015

\begin{abstract}
In order to maximize energy from ambient vibration sources, wide band harvesters working at a range of frequencies are important. This paper presents an electrodynamic energy harvester model working for a frequency band from $25 \mathrm{~Hz}$ to $45 \mathrm{~Hz}$. The developed converter consists of a magnetic spring formed by one moving magnet placed between two fixed magnets. A ring magnet is placed around the moving magnet leading to additional nonlinear stiffness to increase the power output. A comparison to a basic configuration electrodynamic converter was carried out by finite element analysis to show that a significant increase in power output was realized. Simulation results have been confirmed by experimental investigations under harmonic excitations. Based on the experimental time series, we have examined the frequency spectrum and phase portraits to identify the dynamic response of the system. In conclusion, the generator is able to harvest 1.5 times more energy than the simple generator for the bandwidth of $20 \mathrm{~Hz}$ with the resonant frequency of $35 \mathrm{~Hz}$ and the excitation amplitude of $2 \mathrm{~mm}$.
\end{abstract}

\section{Introduction}

Different developed converters were devoted to work for resonant frequency due to the linearity of the system [1-3]. However, in many applications, ambient vibrations have different working frequencies, e.g. industrial machines generate vibrations with a frequency in the range of 10 to $100 \mathrm{~Hz}$ [4]. In order to harvest energy from random excitations, generators should be able to work for wide frequency band instead of resonant one. For that, electrodynamic principle presents one of the most useful methods, which is able to reach the necessary power level in the range of $\mu \mathrm{W}$ at

a e-mail: sonia.bradai@s2013.tu-chemnitz.de 
such excitations, e.g. by using adaptive mechanical or electrical tuning for the working frequency [5-7] or by proposing a bulky generator array [8]. Another approach is to use hybrid systems to realize broadband harvester [9], apply self tuning $[5,10]$ or exposing a piezoelectric converter on nonlinear effects. One of the realization is a piezoelectric beam is used with a ferromagnet [11-16]. By additional magnets in the surrounding of the beam, nonlinear magnetic interactions occur and affect dynamical response of the beam. This allows to get an energy outcome for a broad frequency band. Mann and Sims [17] investigated magnetic levitation system, consisting of the moving magnet working as a mechanical resonator, coils and fixed magnets.

On the other hand, Sato et al. [18] investigated a novel electromagnetic wideband generator. The system consists on the use of magnetic core placed in the coil axis which is fixed and surrounded by moving magnets attached to the end of an cantilever. The presence of the magnetic core leads to generate nonlinearity for the harvester. As result, the generator was able to harvest $0.1 \mathrm{~mW}$ for frequency band of $30 \mathrm{~Hz}$. In all these solutions, the wide frequency band was realized by introducing nonlinearity to the dynamic behavior of the harvester. Stanton et al. [11] and Martens et al. [19] proved that nonlinear behavior could improve the harvester performance for harmonic and random ambient excitations. The analysis of the degree of nonlinearity is decisive for the characterization of the frequency band width.

In this paper, a wideband energy harvester is proposed based on the electrodynamic principle. To evaluate the generator, a finite element analysis is conducted to compare two nonlinear configurations. The harvester is designed and realized in the conditions of an harmonic excitation. The dynamic response of the two configurations is examined based on voltage time series and the energy outcome and the frequency response of the harvester are evaluated.

\section{Finite element analysis}

In this section, finite element analysis is used in order to investigate the magnetic field distribution and its impact on the coil in two nonlinear configurations. The design and the optimization of the wide band harvester in terms of magnet polarization are also investigated.

\subsection{Model description}

A comparison of two nonlinear electrodynamic generators is studied. A 2D axisymmetric finite element model is used since the generators present a cylindrical shape.

The behavior of the two generators is studied based on dynamic analysis. Figure 1 presents the developed finite element model using Comsol Multiphysics for this study. A refined mesh is used in the surrounding of coil/magnets boundary for more accurate results. Based on Faraday's law (Eq. (1)), the energy outcome of the electrodynamic generators depends principally on the magnetic field variation. For that, to compare the two configurations, the distribution of the magnetic field variation through the coil is studied.

$$
e=N \frac{d \Phi}{d t}
$$

where $e$ represents the electromotive force, $N$ is the number of turns of wire and $\Phi$ is the magnetic flux through a single loop.

The two converters are based on the use of magnetic spring principle. For the first one, two magnets are fixed in the bottom and top of the housing and one magnet is 


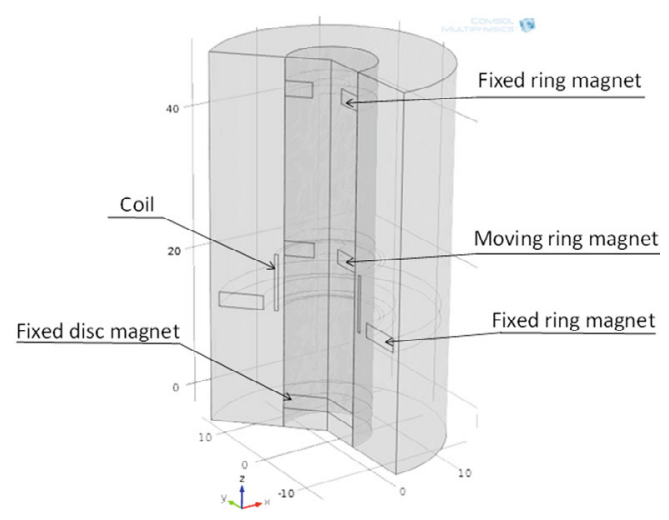

Fig. 1. Developed finite element model.

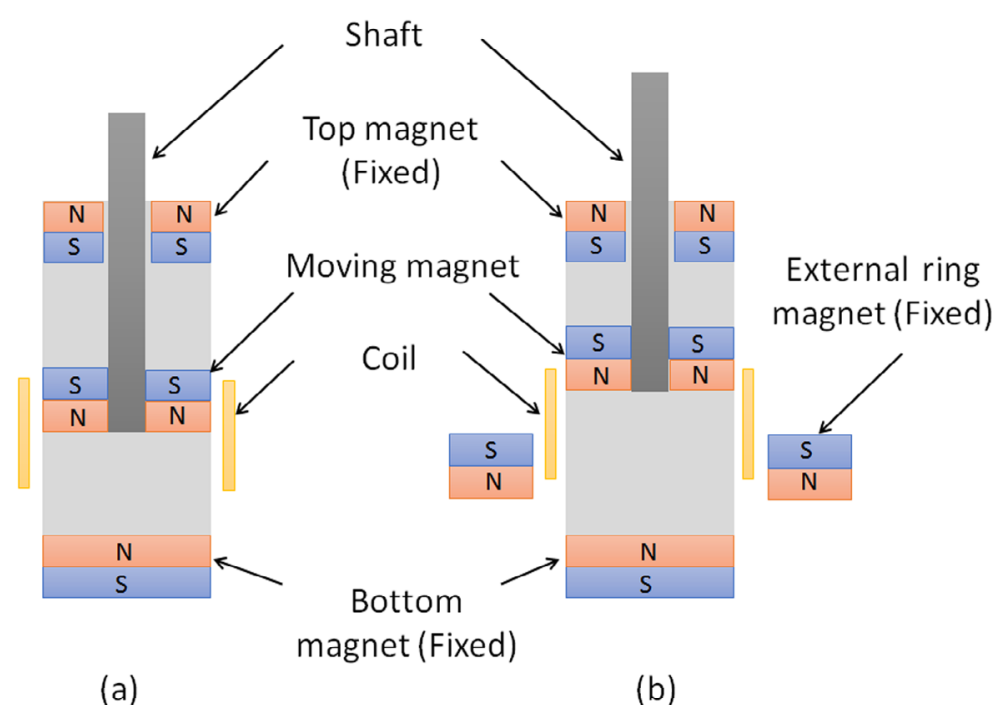

Fig. 2. Schematic for the two configurations (a) without external magnet (b) with external magnet.

moving in between due to the presence of repulsive magnetic force (Fig. 2(a)). For the second generator, an external ring magnet in the surrounding of the moving magnet is added as shown in Fig. 2(b). A fixed coil is placed surrounding the moving magnet from which a generated voltage is harvested. Used magnets and coil characteristics are presented in Table 1.

\subsection{Simulation results}

Since the magnetic field distribution is non-uniform, we propose to evaluate the average magnetic field through the coil. The results are presented for the resonant frequency which is equal to $35 \mathrm{~Hz}$ and for an excitation amplitude of $2 \mathrm{~mm}$ (the acceleration amplitude is $9.86 \mathrm{~g}$ ). A comparison of axial and radial polarization for the ring magnet is studied. Figure 3(a) presents the average magnetic field through the coil versus time. It can be seen that using axial polarization for the ring magnet presents 
Table 1. Parameters for numerical simulation.

\begin{tabular}{l|l}
\hline Parameter & Value \\
\hline Center and Top ring magnet & $12.7 \times 6.4 \times 1.6 \mathrm{~mm}^{3}$ \\
Bottom disc magnet & $14.3 \times 3.2 \mathrm{~mm}^{2}$ \\
External ring magnet & $31 \times 19 \times 2 \mathrm{~mm}^{3}$ \\
Coil turn & 250 turns \\
Coil wire diameter & $0.1 \mathrm{~mm}$ \\
Coil resistance & $22 \Omega$ \\
Coil material & Copper \\
Magnets polarization & Axial \\
Magnets material & NdFeB \\
\hline
\end{tabular}

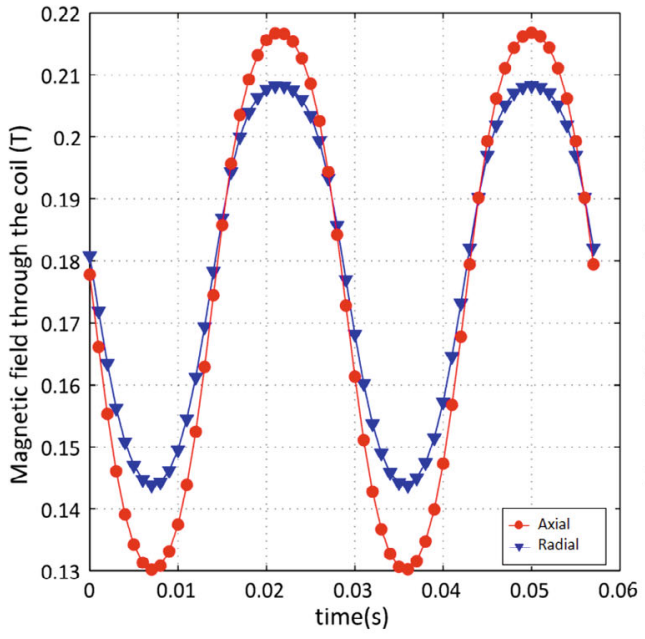

(a)

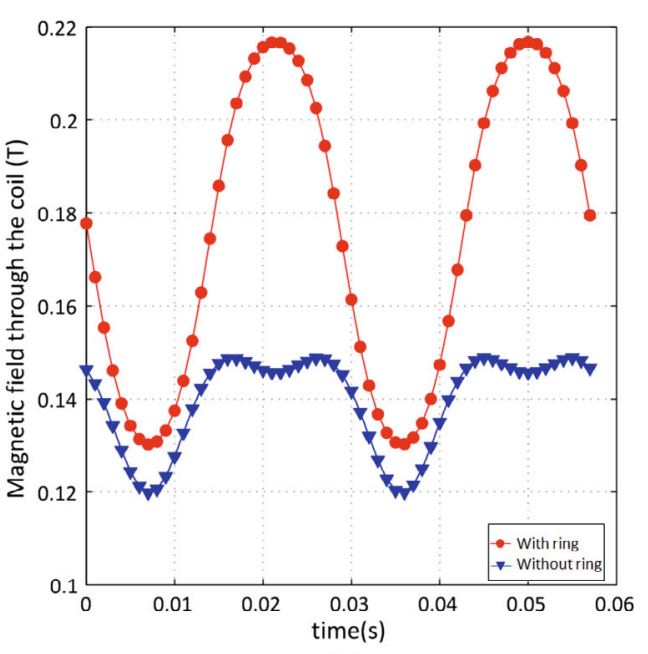

(b)

Fig. 3. Simulated variation of magnetic field through the coil (a) for axial and radial polarization with external ring magnet (b) with and without external ring magnet.

a better variation of the magnetic field which can reach $0.0863 \mathrm{~T}$ instead of $0.0644 \mathrm{~T}$ for radial polarization.

Furthermore investigation, Fig. 3(b) presents the distribution of the magnetic field variation through the coil for both configurations. As it is shown, the variation of the magnetic field adding an external ring magnet is equal to $0.0863 \mathrm{~T}$. In the case of using only a moving disc magnet (Fig. $2(\mathrm{a})$ ), the variation reaches only $0.0285 \mathrm{~T}$. As conclusion, adding external axial ring magnet enables to reach a maximum level of magnetic field variation through the coil. This affects directly the energy outcome of the converter which will be evaluated with the experiment in the following section.

\section{Experimental investigations}

\subsection{Experimental setup}

In this section, a description of the harvester design and experimental results are detailed. For the design, the setup includes a ring magnet which is placed in the top box and a disc magnet placed in the bottom box. A center ring magnet is inserted through the moving axis. A ring magnet is placed in the bottom box in the surrounding of the 


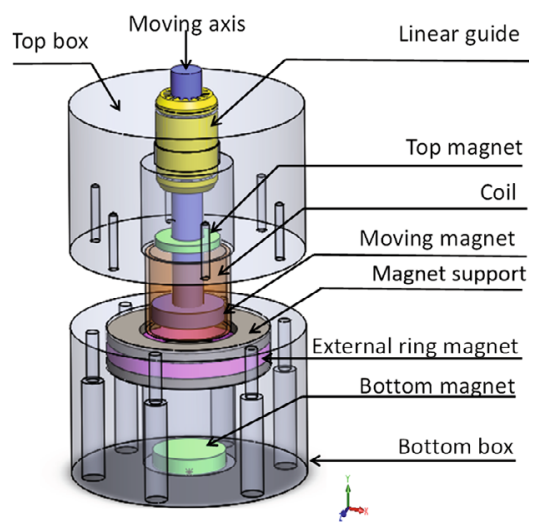

Fig. 4. Designed broadband electrodynamic generator.
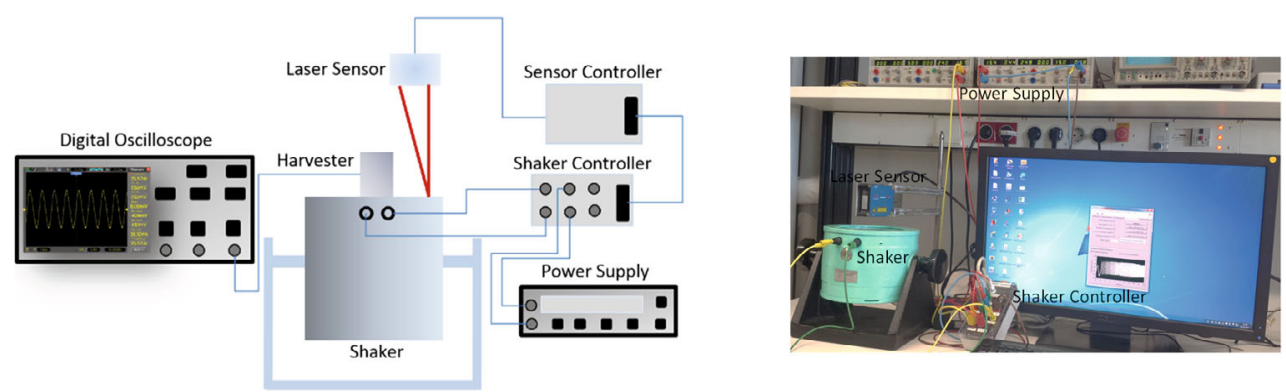

Fig. 5. Experimental setup.

moving ring magnet. Receiving external excitation, the axis and the center magnet are free to move vertically between the two fixed magnets. The magnets are placed in such a way they create repulsive forces. In order to get a bandwidth working generator, external ring magnet is placed surrounding the moving magnet. In between, the coil is placed as shown in Fig. 4. Factors like size and material properties are not taken into consideration since the focus is to enhance the working frequency range of the harvester and not the electrical output energy.

The used experimental setup is illustrated in Fig. 5. It consists on the use of an electrodynamic shaker as an external artificial vibration source controlled by a laser sensor to measure the applied displacement and frequency. Together with the laser sensor, a controller unit and an amplifier, the shaker is operated in a closed loop which allows controlling the vibration amplitude (pure y-direction).

The harvester is mounted on the shaker in such a way that the vibration will be transmitted directly to the moving axis. For the amplitude excitation, it is fixed for all the experiment to $2 \mathrm{~mm}$ and the frequency is varying from 10 to $60 \mathrm{~Hz}$. The acceleration is calculated relative to the frequency and amplitude excitation and it is varying from $0.4 \mathrm{~g}$ to $14 \mathrm{~g}$. The induced voltage was measured through a digital oscilloscope.

\subsection{Evaluation of the output voltage}

Peak to peak open circuit voltage is evaluated under a variation of the excitation frequency in the range of 10 to $60 \mathrm{~Hz}$. As it is shown in Fig. 6(a), the maximum voltage 


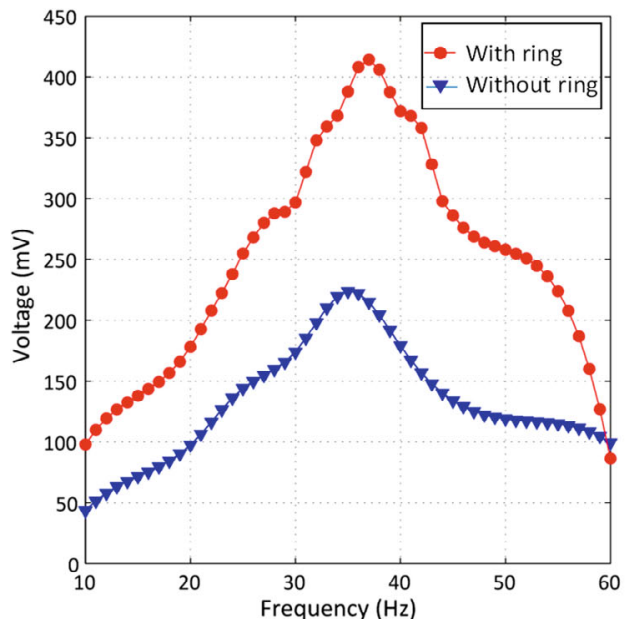

(a)

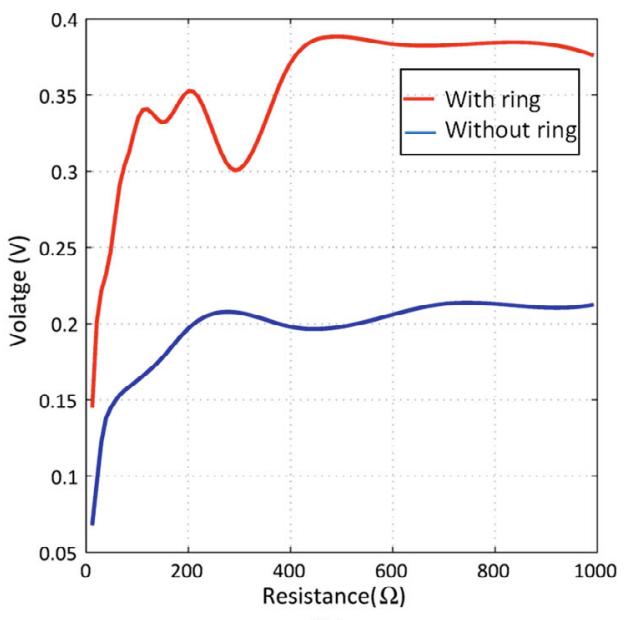

(b)

Fig. 6. (a) Open circuit voltage pk-pk relative to the excitation frequency. (b) Output voltage relative to variable the load resistance at resonant frequency.

for the two systems is reached in the range of $35 \mathrm{~Hz}$. The induced voltage is higher for the generator adding the external ring magnet. This is due to the improvement of the magnetic field variation using external axial ring magnet. The output voltage versus load resistance (from $12 \Omega$ to $1 \mathrm{k} \Omega$ ) and at resonant frequency is presented in Fig. 6(b).

It can be seen that for the configuration without ring magnet, the pk-pk voltage increases with increasing the load resistance from $12 \Omega$ to $240 \Omega$ and then it remains almost constant. For the second configuration, the closed loop pk-pk voltage increases and then decreases before remaining constant for load resistances more than $400 \Omega$. For some load resistance, we remark that the voltage drops and this can be due to the stray capacitance of the coil which is dissipated by increasing the load resistance.

\section{Nonlinear characteristics}

To identify the dynamics of the systems, the frequency spectrum and the phase portraits of the two configurations with loading at resonance are investigated. The voltage time series of experimental results for $35 \mathrm{~Hz}$ excitation frequency for the two configurations are shown in Figs. 7(a) and 7(b). First of all, one should notice that the amplitude of voltage oscillations in the system with the ring magnet (Fig. 7(b)) is higher comparing to the original system without such a magnet (Fig. 7(a)). This is probably caused by stronger magnetic interactions in the configuration with the ring magnet. Additionally, one can see that the case without the ring magnet (Fig. 7(a)) shows less symmetric response comparing to the system with the ring magnet (Fig. $7(\mathrm{c}))$. Figure $7(\mathrm{c})$ and Fig. $7(\mathrm{~d})$ present the corresponding Fourier transforms of the examined measured voltage output. The main peak is related to the driving excitation frequency $(35 \mathrm{~Hz})$.

It can be also seen that the excitation frequency is accompanied by the higher harmonics in both figures. Such harmonics are usually present in nonlinear system responses. The Fourier spectrum in the case of the configuration without the ring 


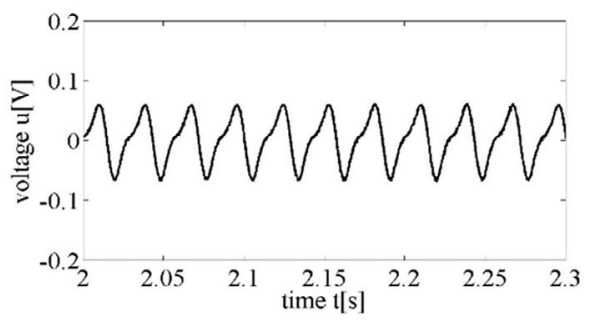

(a)

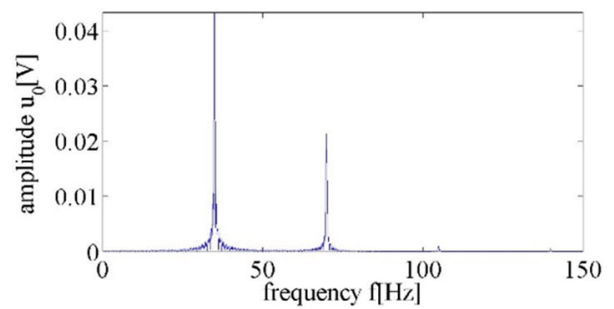

(c)

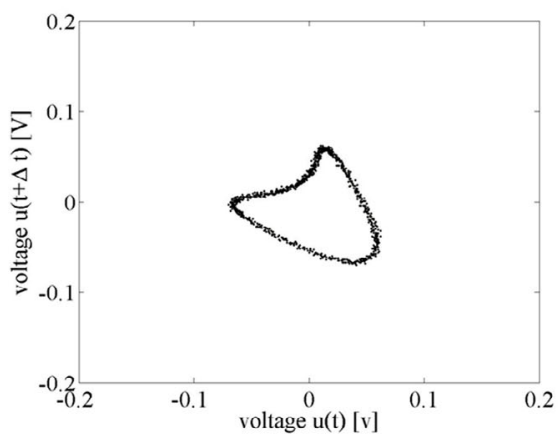

(e)

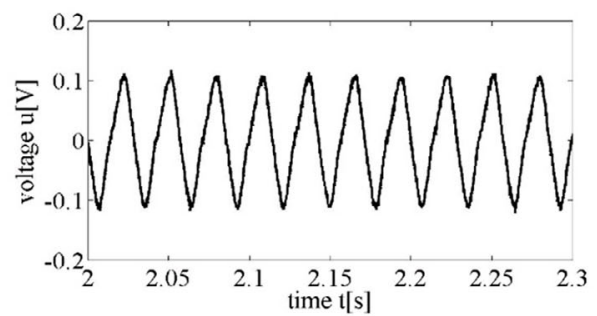

(b)

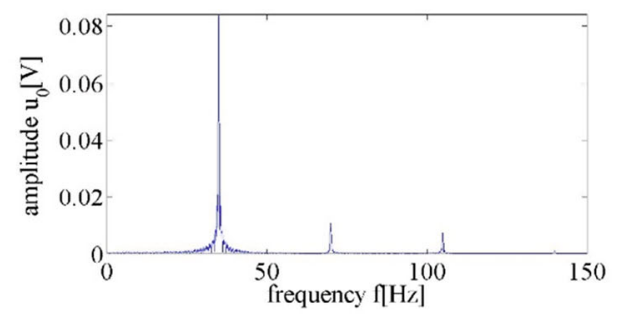

(d)

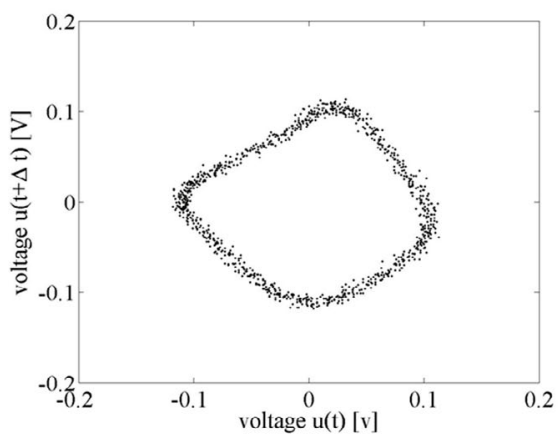

(f)

Fig. 7. Time series (a), (b), Fourier spectrum (c), (d) and phase portraits for the output voltage with loading. The excitation frequency: $35 \mathrm{~Hz}$; cases: without the ring magnet (a), (c), (e) and with the ring magnet (b), (d), (f), respectively.

magnet present one other clear higher harmonics with about $0.02 \mathrm{~V}$ amplitude. In the other side, the configuration with the ring magnet presents two other clear higher harmonics with about $0.02 \mathrm{~V}$ amplitude.

However the heights of the peaks are also important. As the scale of vertical axes are fixed by the height of the main peak, it is worth to notice that in the presence of the ring magnet the additional higher harmonics peaks are relatively smaller. This conclusion is consistent with the magnetic field simulation results (see Fig. 3(b)). In case without the ring magnet there was a visible double harmonic component.

Figure 7(e) and Fig. 7(f) represent the portraits in the reconstructed phase space in the $u(t+\Delta t)$ versus $u(t)$ voltage time series in Fig. 7(a) and Fig. 7(b) respectively. Following Takens [20], the characteristic delay $\Delta t$ can be used to reconstruct the second coordinate. In our case it has been chosen as one quarter of the excitation period. The reconstructions show that in both cases the system is far from the harmonic however the phase portrait with ring magnet (Fig. 7(f)) shows more symmetric convex shape. 


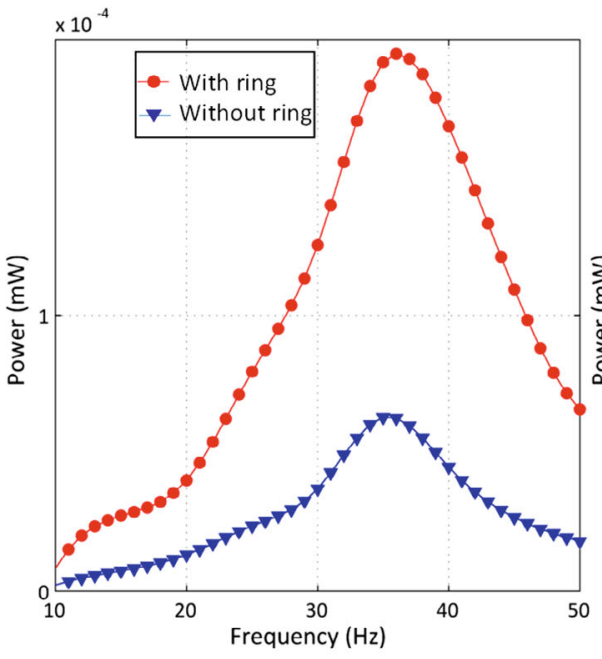

(a)

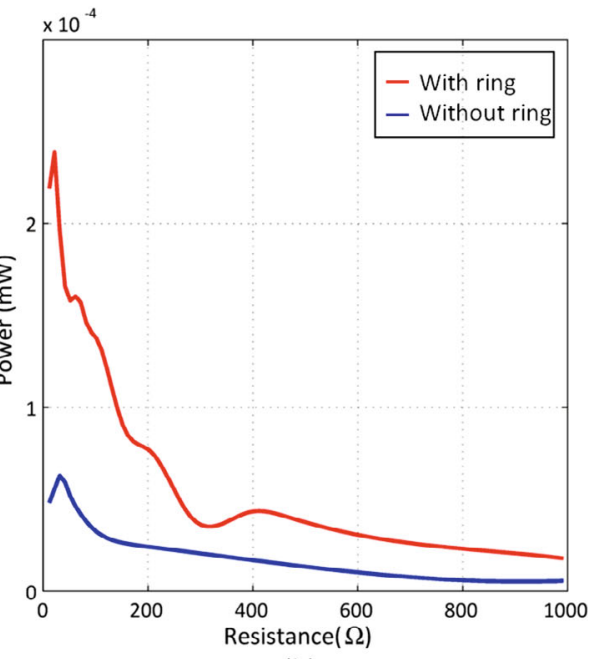

(b)

Fig. 8. (a) Power output relative to the excitation frequency for the optimal load resistance. (b) Power output relative to the load resistance for resonant frequency.

\section{Power output and discussions}

To evaluate the bandwidth, the output power is calculated. Optimal load resistance is determined through the measurement of the output power for variable resistance from $12 \Omega$ to $1 \mathrm{k} \Omega$ and for the resonant frequency $(35 \mathrm{~Hz})$. Figure 8 (a) illustrates that the optimal resistance is equal to $33 \Omega$. In order to evaluate the bandwidth of the two systems, variation of the output power relative to the frequency for the optimal load resistance is presented (Fig. 8(b)). It is clear that adding the ring magnet, the bandwidth is increased from 10 to $20 \mathrm{~Hz}$. Experimental and simulation results confirm that adding the magnet improves the output power level and the bandwidth of the converter. The output power level is not evaluated since the architecture was not optimized. The goal in this study was in particular to evaluate the bandwidth size.

\section{Conclusion}

Harvesting energy from natural vibration source is challenging due to the natural frequency variation. As solution, design of wideband electromagnetic energy harvester is investigated in this work. A comparison of two electromagnetic generators is studied. The proposed concept consists on the use of a ring magnet added in the surrounding of the moving magnet. In our system, we applied harmonic excitation of the moving magnet and the resonating magnetic field inside the coil was caused by the nonlinear interactions between moving and distributed fixed magnets. The harmonic kinematic excitation was applied to the moving magnet. This is a different configuration comparing to Mann and Sims [17] where the excitation was subjected to the casing and the moving magnet played the role of a mechanical resonator. The results show that a bandwidth of $20 \mathrm{~Hz}$ with resonant frequency of $35 \mathrm{~Hz}$ is reachable. Nonlinear behavior was studied through FFT and phase portrait analysis. Surprisingly that analysis confirmed that the system with higher power output looks more linear. The aim of this work was to improve the bandwidth, enhancement for the generator energy outcome is not treated and will be studied in future. 
The authors gratefully acknowledge the financial support from Deutscher Akademischer Austauschdienst (DAAD). Additionally, GL gratefully acknowledges the support of the Polish National Science Center under Grant No. 2012/05/B/ST8/00080.

\section{References}

1. S. Bradai, S. Naifar, T. Keutel, O. Kanoun, Electrodynamic resonant energy harvester for low frequencies and amplitudes, in IEEE International Conference on Instrumentation and Measurement Technology (Montevideo, 2014), p. 1152

2. M. Hendijanizadeh, S.M. Sharkh, S.J. Elliott, M. Moshrefi-Torbati, Smart. Mater. Struct. 22, 125009 (2013)

3. E. Arroyo, A. Badel, F. Formosa, J. Intell. Mater. Syst. Struct. 24, 2023 (2013)

4. K. Najafi, T. Galchev, E.E. Aktakka, R.L. Peterson, J. McCullagh, Microsystems for energy harvesting, in 16th International Conference on Solid-State Sensors, Actuators, and Microsystems (Transducers 2011), (Beijing, China, 2011), p. 1845

5. M. Lallart, S.R. Anton, D.J. Inman, J. Intell. Mater. Syst. Struct. 21, 897 (2010)

6. C. Eichhorn, R. Tchagsim, N. Wilhelm, P. Woias, J. Micromech. Microeng. 21, 104003 (2011)

7. D. Zhu, S. Roberts, T. Mouille, M.J. Tudor, S.P. Beeby, Smart. Mater. Struct. 21, 105039 (2012)

8. D. Zhu, M.J. Tudor, S.P. Beeby, Meas. Sci. Technol. 21, 022001 (2010)

9. S. Mahmoudi, N. Kacem, N. Bouhaddi, Smart. Mater. Struct. 23, 075024 (2014)

10. A. Badel, G. Sebald, D. Guyomar, M. Lallart, E. Lefeuvre, C. Richard, J. Qiu, J. Acous. Soc. Amer. 119, 2815 (2006)

11. S.C. Stanton, C.C. McGehee, B.P. Mann, Appl. Phys. Lett. 95, 174103 (2009)

12. A. Erturk, J. Hoffmann, D.J. Inman, Appl. Phys. Lett. 94, 254102 (2009)

13. F. Cottone, H. Vocca, L. Gammaitoni, Phys. Rev. Lett. 102, 080601 (2009)

14. G. Litak, M.I. Friswell, S. Adhikari, Appl. Physics Lett. 96, 214103 (2010)

15. M. Kim, J. Dugundji, B.L. Wardle, Smart Mater. Struct. 24, 055006 (2015)

16. B. And, S. Baglio, F. Maiorca, C. Trigona, Sensors Actuators A: Phys. 202 (2013)

17. B.P. Mann, N.D. Sims, J. Sound. Vib. 319, 515 (2009)

18. T. Sato, H. Igarashi, Smart Mater. Struct. 24, 025033 (2015)

19. W. Martens, U. von Wagner, G. Litak, Eur. Phys. J. Special Topics 222, 1665 (2013)

20. F. Takens, Detecting Strange Attractors in Turbulence Dynamical Systems and Turbulence (Lecture Notes in Mathematics, 1981) 